\title{
Platelets, eryptosis, amiodarone, aspirin, Artemisia
}

\section{Summary}

Platelets play an important role in malaria. They contribute to the eryptosis of Plasmodium infected erythrocytes and to the phagocytosis of merozoites. Some herbs or drugs like Artemisia, amiodarone, aspirin may interfere with this process, positively or negatively.

\section{Thrombocytopenia}

Thrombocytopenia is a common finding in malaria. Median platelet counts are lower among severe cases than in mild cases, and in children who died than among those who recovered. Infected erythrocytes may adhere to platelets, and the clumps of infected erythrocytes and platelets are associated with severe disease. ${ }^{1}$ The major impact of malaria is on thrombocytes. It is more frequent than anaemia and can be used as a predictor of severity of malaria. A review paper from Morocco studied the main hematological disturbances due to malaria. Thrombocytopenia was found in $90 \%$ of cases, lymphocytopenia in $58 \%$ and anemia only in $23 \%$ of cases. Similar hematological changers were found by several authors. ${ }^{2-12}$ Graphical representation shows it clearly (Figure 1)(Figure 2).

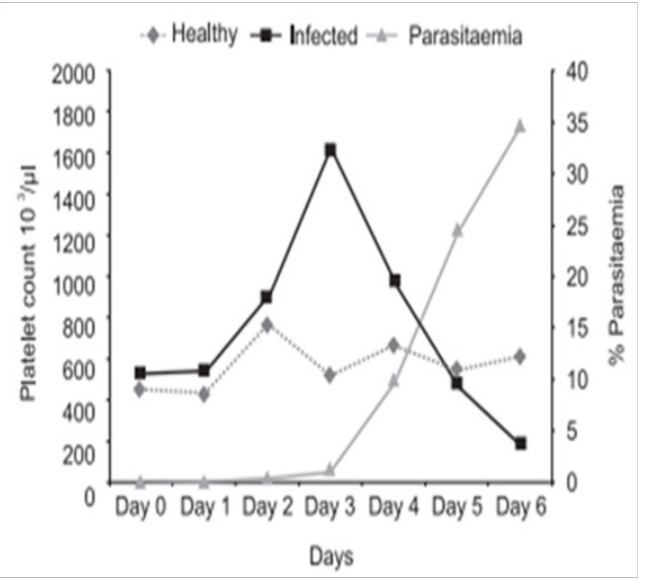

Figure I Platelet count fluctuations associated with $P$. berghei rodent malaria model. $^{13}$

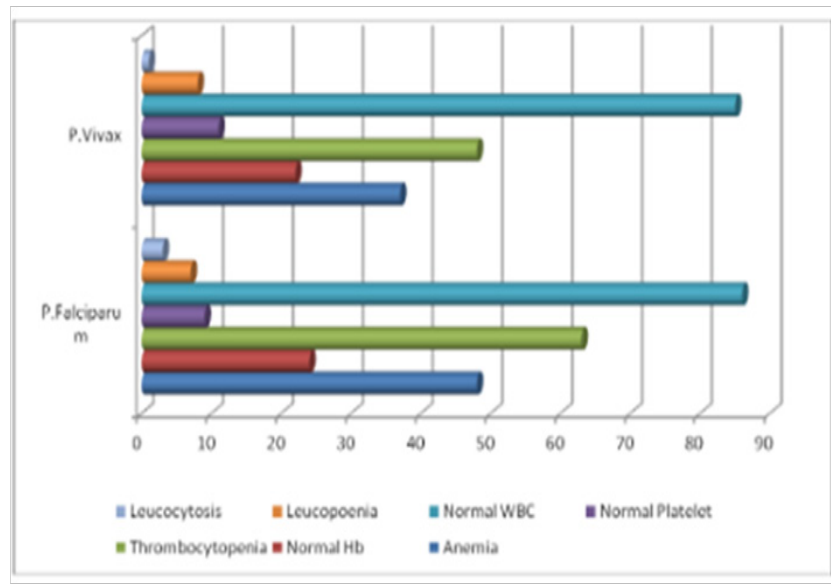

Figure 2 Hematological changes in P. Falciparum and P.Vivax malaria. ${ }^{14}$
Volume 6 Issue 5 - 2018

\author{
Pierre Lutgen,' Jerôme Munyangi \\ 'IFBV-BELHERB, Luxembourg \\ ${ }^{2}$ University of Kolwezi, Democratic Republic of Congo
}

Correspondence: Pierre Lutgen, IFBV-BELHERB, BP 98 L-6905, Niederanven, Luxembourg, Email latgenp@gms.lu

Received: September 17, 2018 | Published: October 15, 2018

But the role of these dramatic changes in platelet count is not well understood and the opinions are controversial. Some claim that platelets kill intraerythrocytic malarial parasites and mediate survival to infection. Platelets bind directly with infected erythrocytes, particularly mature stages. They bind less to uninfected erythrocytes in the bloodstream. Platelet-erythrocyte complexes comprise a major proportion of the total platelet pool in malaria patients and may therefore contribute considerably to malarial thrombocytopenia. Other authors describe platelets as covercytes with pseudopods, rather than phagocytes. ${ }^{15-18} \mathrm{~A}$ quite opposite thesis is presented by another research group, stating that thrombocytopenia correlates with the development of severe falciparum malaria. ${ }^{19}$ Experiments in mice showed that animals genetically deficient in platelets were significantly more susceptible to death from Plasmodium chabaudi infection than were isogenic non-platelet-deficient mice. Similarly, aspirin-treated mice were more susceptible to death from P. chabaudi infection than were placebo-treated animals. ${ }^{20}$

\section{Eryptosis}

Platelets probably play a key role in the eryptosis of Plasmodium infected erythrocytes. Similar to apoptosis of nucleated cells, eryptosis or suicidal erythrocyte death is a physiological mechanism eliminating defective erythrocytes. It is characterized by erythrocyte shrinkage and cell membrane scrambling. Eryptosis is triggered by a wide variety of xenobiotics, others may inhibit it. Eryptosis stimulating xenobiotics may accelerate removal of Plasmodium infected erythrocytes and thus favorably influence the clinical course of malaria. Eryptosis, in malaria, is not restricted to parasitized cells. Eryptosis participates in the innate defense through restriction of intracellular pathogens propagation. In an in vitro culture it was shown that while parasites infected and developed normally in control non-eryptotic erythrocytes, cultures developed with eryptotic erythrocytes had a marked decrease in parasitemia. A great number of free merozoites remained in eryptotic cultures, indicating that these cells were not susceptible to invasion and consequently controlled parasite propagation.

Malaria parasites are dependent on glucose as a nutrient source. As Plasmodia have no capacity to store energy in the form of glycogen, they only rely on an exogenous supply of glucose. The metabolism of the infected erythrocyte uses up to 75 times more glucose than uninfected erythrocytes. Glucose is vital for Plasmodium and the erythrocyte where it took refuge. Glucose metabolism represents a critical physiological program that not only provides energy to the cell 
and the parasite it contains to support proliferation, but also directly modulates signaling pathways of cell death. It is likely that a parasite infected erythrocyte at a given moment has exhausted entirely the exogenous supply of glucose. Hypoglycemia is a common problem in malaria. Platelet counts fall in association with hypoglycemia. A study from Tübingen has shown that platelets adhere to glucose depleted suicidal erythrocytes under flow conditions. This will eliminate eryptotic erythrocytes and the merozoites they contain but may also foster thrombo-occlusion and cerebral malaria. ${ }^{21-26}$

\section{Amiodarone}

The malaria parasite faces a dilemma. It depends on the opening of ion channels in the erythrocyte cell membrane, as they allow the uptake of nutrients, but also $\mathrm{Ca}^{++} \cdot \mathrm{Ca}^{++}$however stimulates eryptosis and simultaneous death of the parasite. The intraerythrocytic parasite deals with this dilemma by delaying the execution of eryptosis by mechanisms well described in the following paper. ${ }^{27}$ Erythrocytes with haemoglobulinopathies or G6PD-deficiency are highly prone to enter eryptosis and this protects the human carrier. Thus, manoeuvers accelerating eryptosis may result in premature eryptosis. Amiodarone which is used to control irregular heartbeat also has a protective effect in malaria. It triggers eryptosis and the clearance of malaria infected erythrocytes. In Plasmodium berghei infected mice amiodarone injections increased the survival. It is interesting to note that amiodarone is also active against other tropical diseases like Chagas or Leishmaniasis, both in vitro and in vivo, probably by disrupting the parasites' $\mathrm{Ca}^{++}$homeostasis. ${ }^{28-31}$

Amiodarone induces hepatocyte microvascular lipid accumulation, and a significant decrease in serum triglycerides and glucose. One of the side effects of amiodarone is the disruption of the hepatic lipid homeostasis. Amiodarone generates lipid containing vacuoles within the hepatocytes. ${ }^{32}$ Very low density lipoproteins (VLDL), are rapidly cleared from plasma and enter hepatocytes. It has been suggested that remnant lipoproteins are initially captured in the space of Disse and that their subsequent internalization into hepatocytes is mediated by members of the LDL-receptor gene family. Similarly to lipoprotein remnants, malaria sporozoites are removed from the blood circulation by the liver within minutes after injection by Anopheles mosquitoes. The sporozoite's surface is covered by the circumsporozoite protein (CSP), and its region II-plus has. Sporozoites, remnant lipoproteins are cleared from the blood compete in vitro and in vivo for binding sites on liver cells. ${ }^{33-35}$ In a previous paper, we described the prophylactic effects on malaria of a ketogenic, high fat diet. ${ }^{36,37}$

Plasmodium needs cholesterol, especially HDL, for its development. After a malaria infection the cholesterol load in the human body diminishes. This is confirmed by many studies and a study from India. The interesting feature of the latter is that they describe accurately that this decrease in total cholesterol has nothing to do with fever per se, but is specifically related to the Plasmodium infection. Very strange is also their finding that only HDL and LDL decrease, but that VLDL sharply increases. Previous studies on the lipid profile in malaria had not noticed this change because they only measured LDL and HDL and ignored VLDL. This VLDL increase is poorly understood. It is possible that at the release of the sporozoites from the liver at the same time VLDL is released. The fact that this increase is noticed on day one of the erythrocyte invasion by merozoites pleads in favor of this hypothesis. ${ }^{38}$

\section{Immunoglobulins}

In malaria endemic areas the population carries a higher level of immunoglobulin antibodies. IgE in association with monocytes or platelets may give rise to reactions that are protective and/ or pathogenic. Most children and adults living in areas where the endemicity of Plasmodium falciparum malaria is high, have significantly elevated levels of both total $\operatorname{IgE}$ antibodies and specific antimalarial IgE bodies in blood. IgE containing immune complexes are known to give rise to monocyte activation via the NO transduction pathway. ${ }^{39}$ Intravenous administration of human immunoglobulin increases platelet counts. The effect is dose dependent and permits to correct thrombocytopenia. The receptor for immunoglobulin on human platelets has been identified. ${ }^{40,41}$ The higher number of $\operatorname{IgE}$ and $\operatorname{IgG}$ antibodies in immune persons is positively correlated with merozoite phagocytosis by monocytes. Platelets opzonised by $\operatorname{IgG}$ activate monocytes to regulatory cells. This activation of monocytes is also seen in leishmaniasis. In mice deficient in platelets the recruitment of monocytes is much slower. ${ }^{42-44}$ Monocytes do ingest merozoites of Plasmodium falciparum, but rarely phagocytose parasitized or nonparasitized erythrocytes. Already in 1981, a French study showed that the monocytes from hyperimmune subjects living continuously in endemic areas were significantly more efficient in the ingestion of merozoites than were those obtained from noninfected subjects or immune individuals who had been away from endemic areas for more than a year. The authors suggest that the role of antibodies can be highly suspected. Similar effects have been observed in rats for the opsonization by antibodies of Plasmodium berghei merozoites (Figure 3). ${ }^{45}$

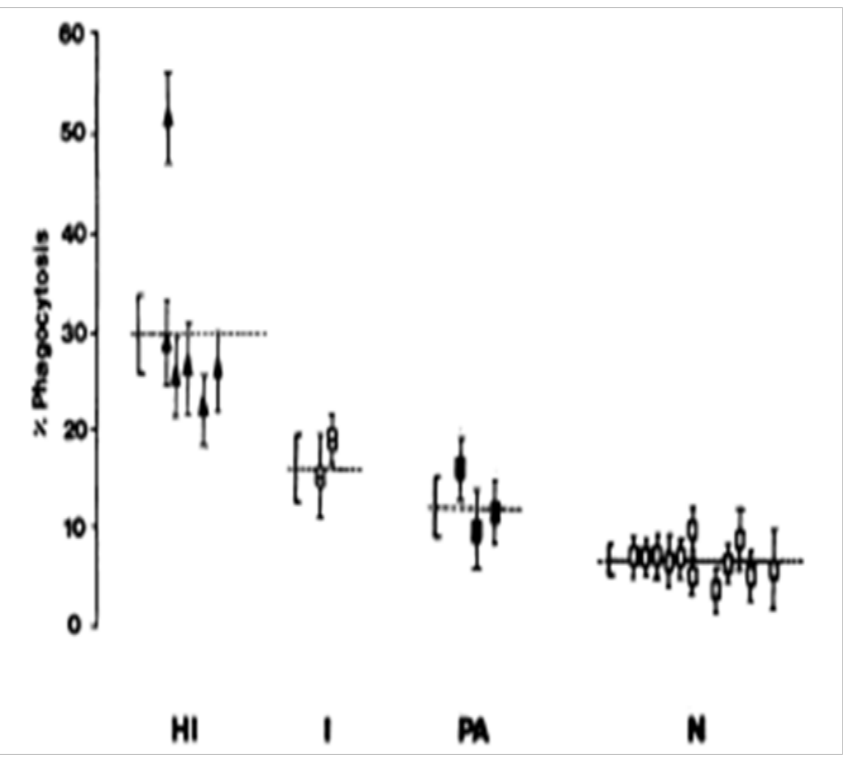

Figure 3 Phagocytosis of P. falcipuram merozoites by monocytes from normal and malarial subjects. ${ }^{46}$

Abbreviations: HI, hyper-immune; I, immune; PA, primary attack; N, normal control.

For IgM the situation is controversial. IgM, the least studied and most enigmatic of the vertebrate Igs, was recently shown to form an intimate relationship with the malaria parasite Plasmodium falciparum. This represents a hitherto unknown immunoevasive mechanism. It was first described in 1972. IgM increases during the 10 first days of the infection and then reverts to the initial values on day 28. Understanding how infected erythrocytes manipulate IgM may also lead to the development of improved therapies. ${ }^{47-49}$

\section{Aspirin}

Aspirin (acetylsalicylic acid) is sold over the counter in Africa and 
appears to be the first line treatment when children or adults have fever. Chronic salicylate poisoning by overdoses is common. Many children brought to a hospital in Africa have a high load of salicylic acid in their blood. Paracetamol is also of common use. In all children admitted to Kilifi District Hospital between July and September 1994, who had a positive blood film for Plasmodium falciparum, and one or more of coma, prostration, or respiratory distress salicylate concentrations were measured. Data were available for 143 children with initial primary diagnoses of severe malaria. 129 had detectable $(>\operatorname{lmg} / \mathrm{dL})$ salicylate. Six of these had salicylate concentrations of $20 \mathrm{mg} / \mathrm{dL}$ or higher. All six had neurological impairment and metabolic acidosis and four were, or became, hypoglycaemic. 21 percent of the mothers gave a dose of aspirin higher than the manufacturer's recommended maximum. These cases suggest that in some children salicylate poisoning may cause or contribute to the development of metabolic acidosis and hypoglycaemia, complications of severe malaria associated with high mortality. ${ }^{50}$ Aspirin, like other platelet antagonists, abrogates the antiparasitic activity of human platelets. Salicylic acid decreases the size of platelets. The Finnish Medical Society guideline on thrombocytopenia states: "Many drugs cause thrombocytopenia relatively frequently... Non-steroidal antiinflammatory drugs, especially acetylsalicylic acid frequently impair platelet function and bring about a bleeding tendency. This tendency is disproportionately strong among thrombocytopenic patients. Aspirin has an effect on the endothelium. This detrimental effect on malaria is known since 40 years and it is unacceptable that WHO has not highlighted this. ${ }^{51-53}$

Aspirin-treated mice are more susceptible to death from Plasmodium chabaudi infection than placebo-treated animals. ${ }^{54}$ Fever is accompanied by glycogen destruction. This was already discovered more than 100 years ago. Glycogen disappears from the liver during tetanus, diphteria and pneumonia. A natural way of our body to fight parasites and diseases. It makes thus complete nonsense to fight fever with aspirin in the early stages of a malaria infection..$^{55}$ Aspirin inhibits cyclooxygenase and affects the TNF- $\alpha$ and prostaglandin E2 produced by the malaria parasite. Let's not forget that only $1 \%$ of those infected by malaria die of the disease because the humane immune system is very efficient. Any interference by xenobiotics may have dramatic consequences. ${ }^{56-58}$

Acidosis and renal failure are an important cause of death in severe malaria. The intake of aspirin before hospitalization plays a role in the development of acidosis and may exacerbate Plasmodium falciparum infection. Paracetamol vs placebo in treatment of nonsevere malaria was studied in Guinea Bissau. It showed that time to parasite clearance is significantly longer in children treated with paracetamol and recrudescence was higher. ${ }^{59}$ Paracetamol by itself may lead to thrombocytopenia. ${ }^{60,61}$

\section{Artemisia plants}

Artemisia plant extracts increase and stabilizes the platelet count in malaria infections and increase survival ( $G$ Bamunuarachchi op.cit.). But this is not the case for artesunate monotherapy where platelets count, hemoglobulin, erythrocytes decrease.${ }^{62}$ But the action is complex. Some of the constituents of Artemisia have opposing roles. Platelet aggregation is induced by arachidonic acid; pentacyclic triterpenes inhibit aggregation. ${ }^{63}$

In a trial in Brazil, at the Campinas university, the Artemisia аппиа tea-taking patients showed shorter bleeding times than those in Coartem group. The bleeding time evaluates the efficiency of the platelets. The patients in the Coartem group presented a mean platelet count much lower than the tea group. Artemisia annua is also a potent inhibitor of TNF- $\alpha$ and PGE2, and may thus reduce the inflammation leading to severe malaria. Probably one of the major reasons why it is important to administer Artemisia annua decoctions during at least seven days to modulate and temper inflammation at the end stage of the malaria infection when most of the platelet population has been deleted. ${ }^{64}$ In vivo studies in Algeria show that Artemisia extracts have strong anti-inflammatory effects. Similar immunomodulatory effects of Artemisia amygdalina were found by a research team in India. This may be important in the trophozoite stage of Plasmodium falciparum infection where inflammation and fever may lead to severe malaria. ${ }^{65-67}$

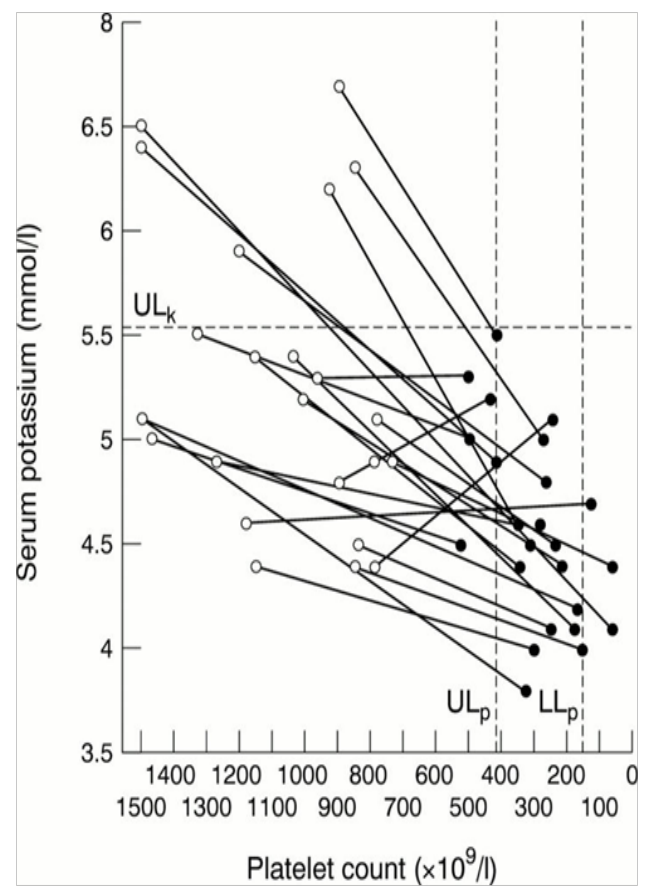

Figure 4 Investigation of possible abnormalities of serum potassium and platelet count in patients with essential thrombocythaemia and significant thrombocytosis. $^{75}$

Artemisia plants are rich in proanthocyanidins, lowering proinflammatory cytokins and TNF- $\alpha .{ }^{68}$ Plasmodium falciparum extensively remodels its host red blood cell. The zeta potential is an electrochemical property of cell surfaces that is determined by the net electrical charge of molecules exposed at the surface of cell membranes. The RBC membrane is negatively charged and is surrounded by a fixed layer of cations. Using an electrophoretic mobility assay, it was found that the main zeta potential was significantly lower in in RBCs infected with Plasmodium falciparum. ${ }^{69}$ At high doses paracetamol can be lethal, but this effect can be reversed by Artemisia absinthium extracts. In a trial on mice acetaminophen produced $100 \%$ mortality at the dose of $1 \mathrm{~g} / \mathrm{kg}$ while pretreatment of animals with plant extract $(500 \mathrm{mg} / \mathrm{kg})$ reduced the death rate to $20 \%$. Pretreatment of rats with plant extract also prevented the acetaminophen induced rise in serum transaminases (GOT and GPT).$^{70}$ Artemisia plants are rich in saponins. They are considered useful in the prophylaxis and treatment of several diseases and are used as adjuvants in malaria vaccines. Saponin from medicinal herbs also activates calcium-dependent potassium channels. This may induce cell scrambling and eryptosis. The 
concentrations required for both eryptosis and hemolysis $(15 \mu \mathrm{g} / \mathrm{ml})$ are easily achieved in vivo. The stimulation of eryptosis is followed by removal of defective erythrocytes prior to hemolysis. By preceding hemolysis, eryptosis protects against the effects of hemolysis. ${ }^{71,72}$ Artemisia annua is also very rich in potassium (Figure 4)..$^{73}$ There is a strong correlation between thrombocytopenia, thrombocythemia, hypokalemia and hyperkalemia. ${ }^{74}$

But the question remains open: does high potassium content in plasma increase the platelet number or is the low potassium content a consequence of a low platelet count. In our humble opinion the first hypothesis merits further studies. The only confirmation we found for this hypothesis in the scientific literature is that Carica papaya, a known antimalarial plant, very rich in potassium, increases the platelet count; in a very significant manner in a in vivo trial in rats, almost doubling it after 7 days administration of an aqueous extract. ${ }^{76}$

\section{Acknowledgements}

None.

\section{Conflict of interest}

The author declares that there is no conflict of interest.

\section{References}

1. Gérardin P, Rogier C, Ka AS, et al. Prognostic value of thrombocytopenia in African children with falciparum malaria. Am J Trop Med Hyg. 2002;66(6):686-691.

2. Khermach A, Khalki H, Louzi L, et al. Perturbations biologiques au cours du paludisme. Pan Afr Med J. 2017;26:174.

3. Abro AH, Ustadi AM. Malria and Hematological changes. Pakistan J of Med Sci. 2008;24(2):287-291.

4. Bhandary N, Shetty V. Thrombocytopenia in malaria: a clinical study. Biomedical Research. 2011;22(4):489-491.

5. Khan SJ, Abbas Y, Ali M. Thrombocytopenia as an indicator of malaria in adult population. Malaria Research and Treatment. 2012;405981:1-4.

6. Lacerda MV, Mourao MP, Santos JB, et al. Thrombocytopenia in malaria: who cares? Mem Inst Oswaldo Cruz. 2011;106(Suppl 1):52-63.

7. Moulin F, Lesage F, Gendrel D. Thrombocytopenia and Plasmodium falciparum malaria in children with different exposures. Arch Dis Child. 2003;88(6):540-541.

8. Ansari S, Khoharo HK, Abro A, et al. Thrombocytopenia in Plasmodium falciparum malaria. J Ayub Med Coll Abbottabad. 2009;21(2):145-147.

9. Senthilkumaar P, Sarojini S. Haematological studies in malaria affected patients in North Chennai, Tamil Nadu. European Journal of Experimental Biology. 2013;3(1):199-205.

10. Kotepui M, Phunphuech B, Phiwklam N, et al. Effect of malarial infection on haematological parameters in population near Thailand-Myanmar border. Malaria J. 2014;13:218.

11. Skudowitz RB, Katz J, Lurie A, et al. Mechanisms of thrombocytopenia in malignant tertian malaria. Br Med J. 1973;2(5865):515-518.

12. Kotepui M, Piwkham D, PhunPhuech B, et al. Effects of Malaria Parasite Density on Blood Cell Parameters. PLoS ONE. 2015;10(3): e0121057.

13. Gayan SB, Ratnasooriya WD, Sirimal P, et al. Artemisia vulgaris L. ethanolic leaf extract reverses thrombocytopenia/ thrombocytosis and averts end-stage disease of experimental severe Plasmodium berghei murine malaria. J Vector Borne Dis. 2014;51:286-293.

14. Sunnaan Amanat, Zafar MH. Pathological Changes of Blood in Malarial Patients. Indo Am J P Sci. 2018;5(6).
15. McMarron BJ, Burgho G, Foote SJ. New insights into the protective power of platelets in malaria infection. Commun Integr Biol. 2013;6(3):e25653.

16. McMorran BJ, Marshall VM, de Graaf C, et al. Platelets kill intraerythrocytic malarial parasites and mediate survival to infection. Science. 2009;323(5915):797-800.

17. Kho S, Barber BE, Johar E. Platelets kill circulating parasites of all major Plasmodium species in human malaria. Blood. 2018;132(12):1332-1344.

18. White JG. Platelets are covercytes, not phagocytes: uptake of bacteria involves channels of the open canalicular system. Platelets. 2005;16(2):121-131.

19. Gramaglia I, Velez J, Combes V, et al. Platelets activate a pathogenic response to blood-stage Plasmodium infection, but not a protective immune response. Blood. 2017;129(12):1669-1679.

20. Morrell CN, Aggrey AA, Chapman LM, et al. Emerging roles for platelets as immune and inflammatory cells. Blood. 2014;123(18):2759-2767.

21. Bruchhaus I, Roeder T, Rennenberg A, et al. Protozoan parasites: programmed cell death as a mechanism of parasitism. Trends Parasitol. 2007;23(8):376-383.

22. Zhao Y, Wieman HL, Jacobs SR, et al. Mechanisms and methods in glucose metabolism and cell death. Methods Enzymol. 2008;442:439-457.

23. Kirk K. Membrane Transport in the Malaria-Infected Erythrocyte. Physiol Rev. 2001;81(2):495-537.

24. Totino PR, Daniel-Ribeiro CT. Refractoriness of eryptotic red blood cells to Plasmodium falciparum infection: a putative host defense mechanism limiting parasitaemia. PLoS One. 2011;6(10):e26575.

25. Walker B, Towhid ST, Schmid E, et al. Dynamic adhesion of eryptotic erythrocytes to immobilized platelets via platelet phosphatidylserine receptors. Am J Physiol Cell Physiol. 2014;306(3):291-297.

26. Hau C Kwaan. Changes in Blood Coagulation, Platelet Function, and Plasminogen-Plasmin System in Diabetes. Diabetes. 1992;41(Suppl 2):32-35.

27. Michael Föller, Diwakar Bobbala, Koka S, et al. Suicide for SurvivalDeath of Infected Erythrocytes as a Host Mechanism to Survive Malaria. Cell Physiol Biochem. 2009;24(3-4):133-140.

28. Bobbala D, Alesutan I, Föller M, et al. Protective effect of amiodarone in malaria. Acta Trop. 2010;116(1):39-44.

29. Piccotti JR, LaGattuta MS, Knight SA, et al. Induction of apoptosis by cationic amphiphilic drugs amiodarone and imipramine. Drugs Chem Toxicol. 2005;28(1):117-133.

30. Benaim G, Sanders JM, Colina C, et al. Amiodarone has intrinsic antiTrypanosoma cruzi activity and acts synergistically with posaconazole. $J$ Med Chem. 2006;49(3):892-899.

31. Gustavo Benaim, Paola Casanova, Suarez Al, et al. Dronedarone, an Amiodarone Analog with Improved Anti-Leishmania mexicana Efficacy. Antimicrob Agents Chemother. 2014;58(4):2295-2303.

32. McCarthy TC1, Pollak PT, Hanniman EA, et al. Disruption of hepatic lipid homeostasis in mice after amiodarone treatment is associated with peroxisome proliferator-activated receptor-alpha target gene activation. $J$ Pharmacol Exp Ther. 2004;311(3):864-873.

33. Sinnis P, Willnow TE, Briones MR, et al. Remnant Lipoproteins Inhibit Malaria Sporozoite Invasion of Hepatocytes. J Exp Med. 1996;184(3):945954.

34. Liane Rabinowich, Oren Shibolet. Drug Induced Steatohepatitis: An Uncommon Culprit of a Common Disease. Biomed Res Int. 2015;168905:1-14.

35. Begriche K, Massart J, Robin MA, et al. Drug-induced toxicity on mitochondria and lipid metabolism: mechanistic diversity and deleterious consequences for the liver. J Hepatol. 2011;54(4):773-794. 
36. Pierre Lutgen. New Insights into Malaria Prophylaxis. Pharm Pharmacol Int J. 2017;5(6):213-217.

37. Pierre Lutgen. Cholesterol, phytosterols and malaria. Malaria world; 2013.

38. Kullu BK, Chakradhar Majhi, Pradhan B, et al. Lipid profiles among Plasmodium falciparum infected, non malarial febrile patients and volunteers. Int J Adv Med. 2018;5(3):556-560.

39. Pierre Lutgen. Antibodies, Prophylaxis, Transmission. Pharm Pharmacol Int J. 2018;6(2):54-56.

40. Hansen RJ, Balthasar J. Effects of intravenous immunoglobulin on platelet count. Blood. 2002;100(6):2087-2093.

41. Manfred Steiner, Ernst F Lischer. Identification of the Immunoglobulin G Receptor of Human Platelets. J Biol Chem. 1986;261(16):7230-7235.

42. Inui M, Tazawa $\mathrm{K}$, Kishi $\mathrm{Y}$, et al. Platelets convert peripheral blood circulating monocytes to regulatory cells via immunoglobulin G. BMC Immunol. 2015;16:20.

43. Awandare GA. Effect of Plasmodium falciparum infection on monocyte and neutrophil function. $\mathrm{PhD}$ thesis biochemistry, University of Ghana; 2001.

44. Goncalves R, Zhang X, Cohen $\mathrm{H}$, et al. Platelet activation attracts a subpopulation of effector monocytes to sites of Leishmania major infection. J Exp Med. 2011;208(6):1253-1265.

45. Shear HL, Nussenzweig RS, Bianco C. Immune phagocytosis in murine malaria. J Exp Med. 1979;149(6):1288-1298.

46. Khusmith S, Druilhe P, Gentilini M. Enhanced Plasmodium falciparum merozoite phagocytosis by monocytes from immune individuals. Infect Immun. 1982;35(3):874-879.

47. Czajkowsky DM, Salanti A, Ditlev SB, et al. IgM, FcmRs and Malarial Immune Evasion. J Immunol. 2010;184(9):4597-4603.

48. Barfod L, Dalgaard MB, Pleman ST, et al. Evasion of immunity to Plasmodium falciparum malaria by IgM masking of protective IgG epitopes in infected infected erythrocyte surface. PNAS. 2011;108(30):1248512490 .

49. Beale PJ, McCormack JD, Oldrey TBN, Thrombocytopenia in malaria with IgM changes. Br Med J. 1972;1(5796):345-349.

50. English M, Marsh V, Amukoye E, et al. Chronic salicylate poisoning and severe malaria. Lancet. 1996;347(9017):1736-1737.

51. Czervionke RL, Hoak JC, Fry GL. Effect of aspirin on thrombin-induced adherence of platelets to cultured cells from the blood vessel wall. J Clin Invest. 1978;62(4):847-856.

52. Fajardo LF. Platelet morphology after aspirin. Am J Clin Pathol. 1975;63(4):554-558.

53. Bose S, Roohi F, Shiralkar M. Effect of variable low doses of aspirin on platelet functions. Indian J Physiol Pharmacol. 1994;38(1):56-60.

54. RT Ellison, McMorran. Platelets Kill Intraerythrocytic Malarial Parasites and Mediate Survival to Infection. Science. 2009;323:797.

55. Lutgen P. Alcohol and Malaria. Pharm Pharmacol Int J. 2018;6(4):310311.

56. Keller CC, Davenport GC, Dickman KR, et al. Suppression of prostaglandins E2 by malaria parasite products and antipyretics promotes overproduction of Tumor Necrosis Factor. J Infect Dis. 2006;193(10):1384-1393.

57. Schrör K. Aspirin and platelets: the antiplatelet action of aspirin and its role in thrombosis treatment and prophylaxis. Semin Thromb Hemost. 1997;23(4):349-356.
58. Kruithof AC, Moerland M, Anastasopoulou EA, et al. Evaluation of the Effect of Aspirin on Platelet Aggregation. J Biomedical Sci Eng. $2015 ; 8(1): 40-45$.

59. Kofoed PE, Ursing J, Rodrigues A, et al. Paracetamol versus placebo in treatment of non-severe malaria in children in Guinea-Bissau: randomized controlled trial. Malaria J. 2011;10:148.

60. Thornton JR, Losowsky MS. Severe thrombocytopenia after paracetamol overdose. Gut. 1990;31(10):1159-1160.

61. Kelton JG, Hirsh J, Carter CJ, et al. Thrombogenic effect of high-dose aspirin in rabbits. Relationship to inhibition of vessel wall synthesis of prostaglandin I2-like activity. J Clin Invest. 1978;62(4):892-895.

62. Bigoniya P, Sahua T, Tiwari V. Hematological and biochemical effects of sub-chronicartesunate exposure in rats. Toxicol Rep. 2015;2:280-288.

63. Babalola IT, Shode FO, Adelakun EA, et al. Platelet-Aggregation Inhibitory Activity of Oleanolic Acid,Ursolic Acid, Betulinic Acid, and Maslinic Acid. Journal of Pharmacognosy and Phytochemistry. 2013;1(6):54-60.

64. Hunt S, Yoshida M, Davis PF, et al. An extract of the medicinal Plant annua modulates production of inflammatory markers. J Inflamm Res. 2015;8:9-14.

65. S Mansour. Evalation de l'effet anti inflammatoire de trois plantes médicinales: Artemisia absinthium, Artemisia herba alba, Hypericum scarboides. Thèse de doctorat Université d'Oran; 2015.

66. Mubashir K, Ganai BA, Khalid G, et al. Evaluation of Artemisia amygdalina D. for Anti-Inflammatory and Immunomodulatory Potential. ISRN Inflammation. 2013;483646:1-5.

67. Bamunuarachchi GS, Ratnasooriya WD, Premakumara S, et al. Artemisia vulgaris L. ethanolic leaf extract reverses thrombocytopenia/ thrombocytosis and averts end-stage disease of experimental severe Plasmodium berghei murine malaria. J Vector Borne Dis. 2014;51(4):286293.

68. Zhang Y, Shi S, Wang W, et al. Antihrombotic effect of grape seed proanthocyanicidins extract in a rat model of deep vein thrombosis. J Vasc Surg. 2011;53(3):743-753.

69. Tokumasu F, Ostera GR, Amaratunga C, et al. Modifications in Erythrocyte Membrane Zeta Potential by Plasmodium falciparum Infection. Exp Parasitol. 2012;131(2):245-251.

70. Gilani AH, Janbaz KH. Preventive and curative effects of Artemisia absinthium on acetaminophen and CCl4-induced hepatotoxicity. Gen Pharmacol. 1995;26(2):309-315.

71. Bissinger R, Modicano P, Alzoubi K, et al. Effect of saponin on erythrocytes. Int J Hematol. 2014;100(1):51-59.

72. McManus OB, Harris GH, Giangiacomo KM, et al. An activator of calcium-dependent potassium channels isolated from a medicinal herb. Biochemistry. 1993;32(24):6128-6133.

73. Pierre Lutgen. New approaches in malaria prophylaxis: endophytic fungi, asparaginase, potassium and papaya. Pharm Pharmacol Int $J$ 2018;6(4):274-276.

74. Graber M, Subramani K, Corish D, et al. Thrombocytosis Elevates Serum Potassium. Am J Kidney Dis. 1988;12(2):116-120.

75. Howard M, Ashwell S, Bond LR, et al. Artefactual serum hyperkalaemia and hypercalcaemia in essential thrombocythaemia. $J$ Clin Pathol. 2000;53(2):105-109.

76. Patil S, Shetty S, Rama B, et al. Evaluation of platelet augmentation activity of Carica papaya leaf aqueous extract in rats. Journal of Pharmacognosy and Phytochemistry. 2013;1(5):57-60. 